\title{
Relativistic electron pitch-angle scattering by electromagnetic ion cyclotron waves during geomagnetic storms
}

\author{
Danny Summers \\ Department of Mathematics and Statistics, Memorial University of Newfoundland, St. John's, Newfoundland, Canada
}

Richard M. Thorne

Department of Atmospheric Sciences, University of California, Los Angeles, California, USA

Received 16 May 2002; revised 13 August 2002; accepted 18 September 2002; published 3 April 2003.

[1] During magnetic storms, relativistic electrons execute nearly circular orbits about the Earth and traverse a spatially confined zone within the duskside plasmapause where electromagnetic ion cyclotron (EMIC) waves are preferentially excited. We examine the mechanism of electron pitch-angle diffusion by gyroresonant interaction with EMIC waves as a cause of relativistic electron precipitation loss from the outer radiation belt. Detailed calculations are carried out of electron cyclotron resonant pitch-angle diffusion coefficients $D_{\alpha \alpha}$ for EMIC waves in a multi-ion $\left(\mathrm{H}^{+}, \mathrm{He}^{+}, \mathrm{O}^{+}\right)$plasma. A simple functional form for $D_{\alpha \alpha}$ is used, based on quasi-linear theory that is valid for parallelpropagating, small-amplitude electromagnetic waves of general spectral density. For typical observed EMIC wave amplitudes $(1-10 n T)$, the rates of resonant pitch-angle diffusion are close to the limit of "strong" diffusion, leading to intense electron precipitation. In order for gyroresonance to take place, electrons must possess a minimum kinetic energy $E_{\min }$ which depends on the value of the ratio (electron plasma frequency/ electron gyrofrequency); $E_{\min }$ also depends on the properties of the EMIC wave spectrum and the ion composition. Geophysically interesting scattering, with $E_{\min }$ comparable to $1 \mathrm{MeV}$, can only occur in regions where (electron plasma frequency/electron gyrofrequency) $\geq 10$, which typically occurs within the duskside plasmapause. Under such conditions, electrons with energy $\geq 1 \mathrm{MeV}$ can be removed from the outer radiation belt by EMIC wave scattering during a magnetic storm over a time-scale of several hours to a day. INDEX TERMS: 2716 Magnetospheric Physics: Energetic particles, precipitating; 2772

Magnetospheric Physics: Plasma waves and instabilities; 2471 Ionosphere: Plasma waves and instabilities; 2730 Magnetospheric Physics: Magnetosphere_inner; KEYWORDS: relativistic electrons, magnetic storms, EMIC waves, electron precipitation, outer radiation belt, strong diffusion scattering

Citation: Summers, D., and R. M. Thorne, Relativistic electron pitch-angle scattering by electromagnetic ion cyclotron waves during geomagnetic storms, J. Geophys. Res., 108(A4), 1143, doi:10.1029/2002JA009489, 2003.

\section{Introduction}

[2] Observations of electromagnetic ion cyclotron (EMIC) waves in the frequency range of $0.1-5.0 \mathrm{~Hz}$ in the Earth's magnetosphere have been reported in a number of studies, e.g., see Anderson et al. [1992a, 1992b], Fraser et al. [1992, 1996], Bräysy et al. [1998], Erlandson and Ukhorskiy [2001], and Fraser and Nguyen [2001]. A main source of free energy for the excitation of magnetospheric EMIC waves is the anisotropic distribution of ring current hydrogen $\left(\mathrm{H}^{+}\right)$ions produced by Earthward ion convection from the magnetotail during magnetic storms or substorms [e.g., Cornwall et al., 1970; Kozyra et al., 1997; Jordanova et al., 2001]. EMIC waves are preferentially excited in a spatially localized zone along the high-density duskside plasmapause, as a result of reduced resonant energy [Corn-

Copyright 2003 by the American Geophysical Union. 0148-0227/03/2002JA009489 wall et al., 1970; Perraut et al., 1976; Horne and Thorne, 1993, 1994] and wave guiding by steep density gradients near the plasmapause [Thorne and Horne, 1997; Kozyra et al., 1997; Jordanova et al., 1998, 2001]. Although EMIC waves are present even during relatively quiet geomagnetic conditions, the waves occur most frequently and are most intense during magnetic storms [Bräysy et al., 1998; Erlandson and Ukhorskiy, 2001], with typical wideband wave amplitudes in the range $1-10 n T$. EMIC waves occur in three distinct bands below the hydrogen $\left(\mathrm{H}^{+}\right)$, helium $\left(\mathrm{He}^{+}\right)$, and oxygen $\left(\mathrm{O}^{+}\right)$ion gyrofrequencies, the excited band(s) being controlled by the ion composition [e.g., Horne and Thorne, 1994], the level of geomagnetic activity [Bräysy et al., 1998], and the location relative to the plasmapause [Fraser and Nguyen, 2001].

[3] Early theoretical studies by Thorne and Kennel [1971] and Lyons and Thorne [1972] considered the process of resonant pitch-angle scattering by EMIC waves in a simple $H^{+}$, e plasma for inducing relativistic electron pre- 
cipitation during the main phase of a magnetic storm. Such gyroresonant pitch-angle scattering was also discussed as a potential mechanism for intense relativistic electron precipitation from the Earth's outer radiation belts [Thorne, 1974; Thorne and Andreoli, 1980]. More recently, using data from balloon-borne X-ray instruments, Foat et al. [1998], Lorentzen et al. [2000], and Millan [2002] have reported terrestrial X-ray bursts from the duskside region of the outer magnetosphere, consistent with bremsstrahlung emission from highly energetic $(\geq 1.0 \mathrm{MeV})$ precipitating electrons. These X-ray observations have been interpreted as an energy selective precipitation of ambient relativistic electrons due to pitch-angle scattering by gyroresonance with EMIC waves. Horne and Thorne [1998] calculated minimum electron energies for cyclotron resonant interaction with various electromagnetic wave modes. In particular, Horne and Thorne [1998] calculated the minimum electron energies for cyclotron resonance with EMIC waves in a multi-ion $\left(\mathrm{H}^{+}, \mathrm{He}^{+}, \mathrm{O}^{+}\right)$plasma for stormtime conditions both inside and outside the plasmapause. Summers et al. [1998, 2001] developed a relativistic theory of wave-particle gyroresonant diffusion for application to the magnetosphere. Summers et al. [1998] demonstrated that electrons in gyroresonance with EMIC waves are subject essentially to pure pitch-angle diffusion.

[4] Satellite observations have revealed that the flux of relativistic $(\geq 1.0 \mathrm{MeV})$ electrons in the outer radiation belt $(3<L<7)$ generally decreases rapidly during the (several hour) onset phase of a magnetic storm. Approximately $25 \%$ of storms result in a net loss of the relativistic electron population (G. D. Reeves et al., Acceleration and loss of relativistic electrons during geomagnetic storms, submitted to Geophysical Research Letters, 2003), while 50\% of storms exhibit a gradual recovery over a few days, often to flux values exceeding the prestorm level by as much as two orders of magnitude. To account for the recovery phase increase, Summers et al. [1998] proposed a model, further developed by Summers and Ma [2000a] that incorporates rapid electron pitch-angle scattering induced by resonant interaction with EMIC waves near the duskside plasmapause and electron stochastic acceleration by cyclotron resonance with whistlermode chorus outside the plasmasphere. In the present paper, we reexamine the mechanism of pitch-angle scattering of electrons by cyclotron resonance with EMIC waves and quantify the efficiency of the mechanism for inducing relativistic electron precipitation losses in a multi-ion plasma during the various stages of a magnetic storm. As well as stochastic acceleration by whistler-mode turbulence, other physical mechanisms have been proposed for explaining the generation of stormtime relativistic electrons, though discussion of these is beyond the scope of the present paper. For descriptions of other proposed acceleration mechanisms and discussion of the associated issues, the reader is referred to $L i$ et al. [1993, 1997], Elkington et al. [1999], Summers and Ma [2000a, 2000b], Hudson et al. [2001], Li and Temerin [2001], Friedel et al. [2002], and Meredith et al. [2002]. The point we emphasize here is that most published models of stormtime electron acceleration do not include electron losses. We contend that a quantitative treatment accounting for the processes governing relativistic electron losses from the radiation belts via precipitation is fundamental to understanding radiation belt dynamics, particularly at relativistic energies. In addition, precipitating relativistic electrons act as a coupling mechanism between the magnetosphere and the Earth's middle atmosphere and can alter the chemical composition of the stratosphere and mesosphere [e.g., Thorne, 1977; Callis et al., 1998]. Relativistic electron precipitation might therefore provide an important Sun-geospace link connecting solar activity with global climate variability.

[5] In this paper we carry out detailed calculations of rates of pitch-angle diffusion $D_{\alpha \alpha}$ due to electron cyclotron resonance with EMIC waves under magnetic storm conditions. In the following section we present the dispersion relations for EMIC waves in a multi-ion (hydrogen, helium, oxygen) plasma, and, for comparison, in a simple electronproton plasma. The relativistic Doppler condition for gyroresonant interaction between electrons and electromagnetic waves is given in section 3, and minimum kinetic energy curves for electrons in resonance with EMIC waves are constructed for varying plasma parameters and ion composition. In section 4 we derive a simple generic formula for the rate of pitch-angle diffusion $D_{\alpha \alpha}$ for electrons in resonance with parallel-propagating (R-mode or L-mode) electromagnetic waves of arbitrary spectral density. The formula is applied to (L-mode) EMIC waves with a Gaussian frequency spectrum and specified by the dispersion relations given in section 2. Plots of the corresponding pitch-angle diffusion coefficient $D_{\alpha \alpha}$ are presented in section 5 for representative plasma parameters. The pitch-angle diffusion coefficients $D_{\alpha \alpha}$ are found to depend strongly on the spectral properties of the waves and on the ion composition, as well as the local plasma density and magnetic field. For EMIC waves with amplitudes $\geq 1 n T$ the rates of relativistic electron pitch-angle diffusion approach the strong diffusion limit, as defined by Kennel and Petschek [1966]. Such scattering results in intense and spatially confined relativistic electron precipitation. Significant loss can occur over many electron drift orbits or several hours. In section 6 , as well as commenting on our conclusions, we briefly consider limitations of the present study. We also point to the need for future studies to quantify more accurately the important process of pitch-angle scattering of relativistic electrons by EMIC waves.

\section{Dispersion Relations}

[6] The linear theory of electromagnetic waves in a cold, uniform plasma is given in standard texts [e.g., Stix, 1992]. The dispersion relation for (L-mode) EMIC waves propagating parallel to a (uniform) background magnetic field in a multi-ion $\left(\mathrm{H}^{+}, \mathrm{He}^{+}, \mathrm{O}^{+}\right)$plasma is

$$
\frac{c^{2} k^{2}}{\omega^{2}}=1-\frac{\omega_{p e}^{2}}{\omega\left(\omega+\left|\Omega_{e}\right|\right)}-\sum_{j=1}^{3} \frac{\omega_{p j}^{2}}{\omega\left(\omega-\Omega_{j}\right)},
$$

where the suffix $j$ denotes the ion species; the values $j=1$, 2, 3 refer to hydrogen $\left(\mathrm{H}^{+}\right)$, helium $\left(\mathrm{He}^{+}\right)$, and oxygen $\left(\mathrm{O}^{+}\right)$, respectively. Here, $\omega$ is the wave frequency, $k$ is the wave number in the direction of propagation, and $c$ is the speed of light; $\left|\Omega_{e}\right|=e B_{0} /\left(m_{e} c\right)$ is the electron gyrofrequency, and $\omega_{p e}=\left(4 \pi N_{0} e^{2} / m_{e}\right)^{1 / 2}$ is the electron plasma frequency, where $B_{0}$ is the magnetic field strength, $e$ is the electron charge, $m_{e}$ is the electron rest mass, and $N_{0}$ is the electron 


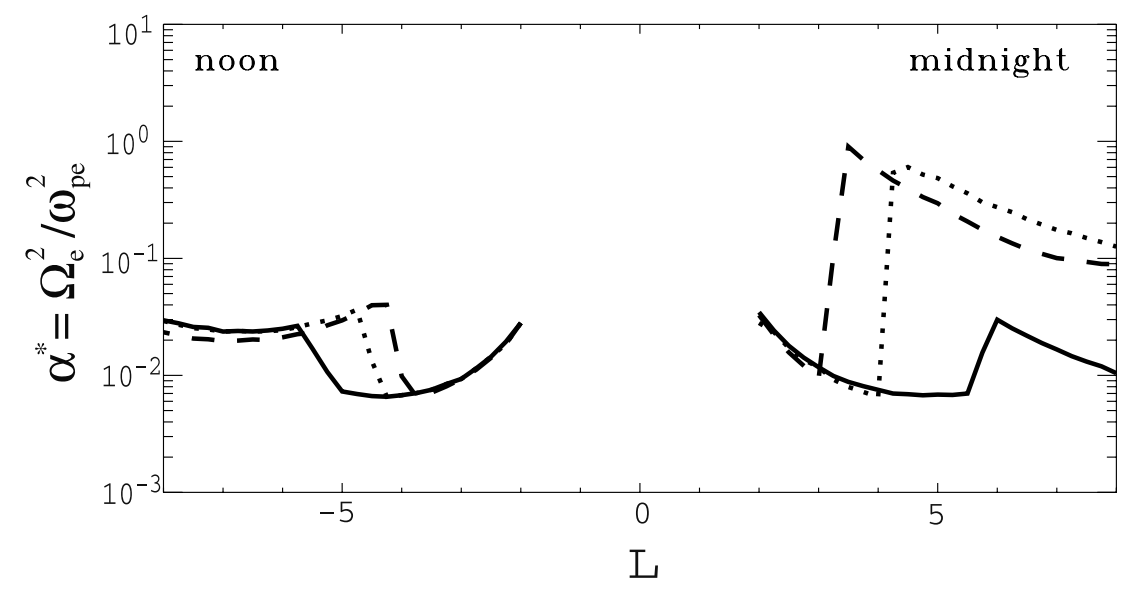

Figure 1. Profiles of the plasma parameter $\alpha^{*}=\Omega_{e}^{2} / \omega_{p e}^{2}$ versus geocentric radial distance along the solar magnetic $x$-axis, constructed from empirical data. The solid, dotted, and dashed curves represent successively higher levels of geomagnetic activity for $K p=1,3$, and 5, respectively. The minimum value of $\alpha^{*}$, which characterizes the plasmapause, is important to the present study.

number density; $\Omega_{j}=q_{j} B_{0} /\left(m_{j} c\right)$ is the ion gyrofrequency and $\omega_{p j}=\left(4 \pi N_{j} q_{j}^{2} / m_{j}\right)^{1 / 2}$ is the ion plasma frequency, where $q_{1}=q_{2}=q_{3}=e, m_{1}=m_{p}, m_{2}=4 m_{p}$, and $m_{3}=16 m_{p}$, with $m_{p}$ $m_{p}$ the proton rest mass. The ion number densities $N_{1}, N_{2}$, $N_{3}$ satisfy the condition $N_{1}+N_{2}+N_{3}=N_{0}$, by assumption of plasma charge neutrality. Equation (1) can be written in the nondimensional form

$$
\frac{1}{u^{2}}=1-\frac{1}{\alpha^{*} \epsilon x}\left(\frac{1}{1+\epsilon x}+\frac{\eta_{1}}{x-1}+\frac{\eta_{2}}{4 x-1}+\frac{\eta_{3}}{16 x-1},\right)
$$

where $u=\omega /(k c)$ is the dimensionless wave phase speed and $x=\omega / \Omega_{1}$ the dimensionless wave frequency, with $\Omega_{1}=\Omega_{H^{+}}$ the proton gyrofrequency; and $\epsilon=m_{e} / m_{p}$. The fractional composition of the ion species, given by $\eta_{1}=N_{1} / N_{0}, \eta_{2}=$ $N_{2} / N_{0}, \eta_{3}=N_{3} / N_{0}$, is such that $\eta_{1}+\eta_{2}+\eta_{3}=1$. In equation (2) we have introduced the nondimensional parameter

$$
\alpha^{*}=\frac{\Omega_{e}^{2}}{\omega_{p e}^{2}}=\frac{1}{\epsilon} \frac{V_{A}^{2}}{c^{2}}=\frac{B_{0}^{2}}{4 \pi N_{0}} \cdot \frac{1}{m_{e} c^{2}},
$$

where $B_{0}^{2} /\left(8 \pi N_{0}\right)$ can be regarded as the magnetic energy per particle, and $V_{A}=B_{0} /\left(4 \pi N_{0} m_{p}\right)^{1 / 2}$ is the Alfven speed for an electron-proton plasma. The dispersion relation corresponding to the special case of an electron-proton plasma is obtained from equation (2) by setting $\eta_{1}=1$ and $\eta_{2}=\eta_{3}=0$; the result is

$$
u^{2}=\frac{\alpha^{*} \epsilon(1+\epsilon x)(1-x)}{\alpha^{*} \epsilon(1+\epsilon x)(1-x)+1+\epsilon} .
$$

[7] The parameter $\alpha^{*}$, which plays an important role in cold plasma theory, depends on the background magnetic field strength $B_{0}$ and the electron number density $N_{0}$. Figure 1 shows the variation of $\alpha^{*}$ in the Earth's inner magnetosphere, against geocentric distance $L=r / R_{E}$ in the equatorial plane, with the dayside to the left. The figure was constructed by using electron number densities from the global core plasma model of Gallagher et al. [2000] and magnetic field values from a modified version T89c of the Tsyganenko [1989] magnetic field model. The solid, dotted, and dashed curves correspond to the respective values of the geomagnetic activity index $K p=1,3$, and 5 . These curves demonstrate the well-known contraction in size of the plasmasphere with increasing magnetic activity. The magnetic local time asymmetry of the plasmapause and the formation of an asymmetric ring current (which lowers the value of $B_{0}$ ) during storm conditions can lead to lower $\alpha^{*}$ values in the duskside plasmapause compared with quiettime values. We therefore consider the range $10^{-3} \leq \alpha^{*} \leq$ $10^{-2}$ to be representative of $\alpha^{*}$-values in or near the equatorial plane, just inside the Earth's plasmapause, or within detached high-density plasma regions.

[8] In Figure 2 we present the EMIC wave dispersion curves (2) for an ion composition $\eta_{1}=0.75, \eta_{2}=0.2, \eta_{3}=$ 0.05 , and the two cases $\alpha^{*}=10^{-3}, 10^{-2} ; u^{2}$ is plotted against $\omega / \Omega_{3}=\omega / \Omega_{O^{+}}=16 x$. We shall refer to the three illustrated wave bands, defined over the respective frequency ranges $0<\omega<\Omega_{\mathrm{O}^{+}}, \Omega_{\mathrm{He}^{+}}<\omega<\Omega_{\mathrm{He}^{+}}$, and $\omega_{\mathrm{H}^{+}}<$ $\omega<\Omega_{H^{+}}$, as the oxygen, helium, and hydrogen bands. The frequencies $\omega_{H e^{+}}$and $\omega_{H^{+}}$are the respective cut-off frequencies for helium and hydrogen, defined as the frequencies where the refractive index $n(=1 / u)$ vanishes. Fieldaligned wave propagation is not possible in the stop-bands specified by $\Omega_{O^{+}} \leq \omega \leq \omega_{H e^{+}}$and $\Omega_{H^{+}} \leq \omega \leq \omega_{H^{+}}$. Comparison of the upper and lower panels of Figure 2 reveals a general reduction in the wave phase speed $u$ as the value of the parameter $\alpha^{*}$ is reduced. This is significant with respect to the calculation of minimum resonant energies, as we discuss in the following section.

\section{Electron Resonant Energy}

[9] The relativistic Doppler condition for gyroresonance between electrons and electromagnetic waves of frequency $\omega$ and parallel wave number $k$ is

$$
\omega-k v_{\|}=N\left|\Omega_{e}\right| / \gamma
$$

where $\gamma=\left(1-v^{2} / c^{2}\right)^{-1 / 2}$ is the Lorentz factor, $v=\left(v_{\|}^{2}+\right.$ $\left.v_{\perp}^{2}\right)^{1 / 2}$ is the electron speed, and $v_{\|}$and $v_{\perp}$ are the electron velocity components in the plasma frame parallel and perpendicular to the ambient magnetic field, respectively; $N$ 

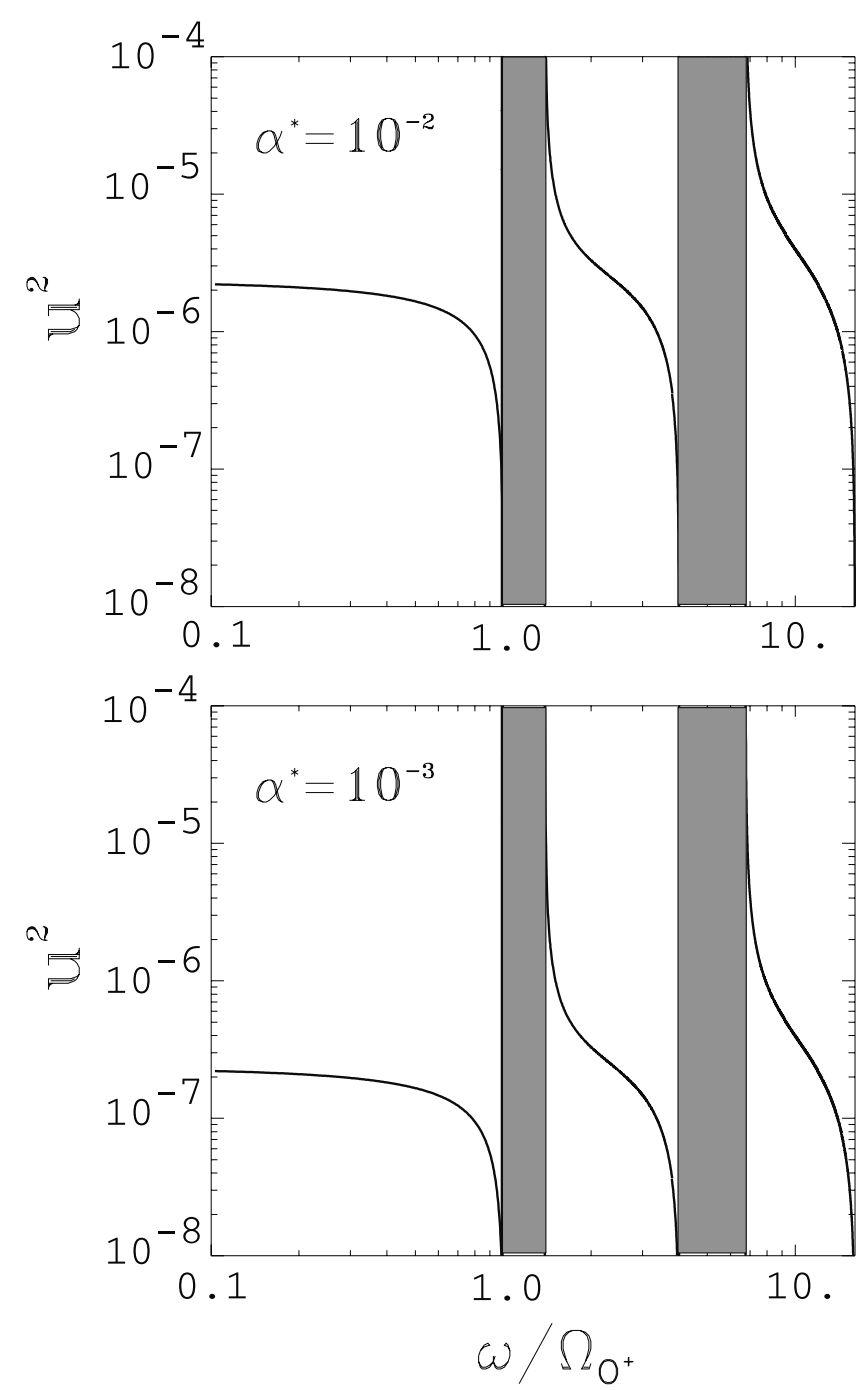

Figure 2. Dispersion curves, given by equation (2), for EMIC waves in a multi-ion plasma, $u^{2}=\omega^{2} /(k c)^{2}$ plotted against the wave frequency $\omega$ in units of the oxygen ion gyrofrequency $\Omega_{O^{+}}$. The hydrogen $\left(\mathrm{H}^{+}\right)$, helium $\left(\mathrm{He}^{+}\right)$, and oxygen $\left(\mathrm{O}^{+}\right)$fractional ion number densities are respectively $\eta_{1}=0.75, \eta_{2}=0.2$, and $\eta_{3}=0.05$.

is the cyclotron harmonic which, in principle, takes on all integer values $(0, \pm 1, \pm 2, \ldots)$. Since we are here considering parallel propagating L-mode EMIC waves, only the value $N=-1$ is relevant. Points $\left(v_{\|}, v_{\perp}\right)$ satisfying condition (5), for a given frequency $\omega$, can be plotted in velocity $\left(v_{\|}, v_{\perp}\right)$ space, and are found to lie on part of a semiellipse in the upper half-plane $\left(v_{\perp} \geq 0\right)$. Thus an electron will be in resonance with a given wave if its velocity components lie on this "resonance ellipse." In general, the kinetic energy $E_{k}$ of an electron in units of its rest energy $m_{e} c^{2}$ is $E=E_{k} /$ $\left(m_{e} c^{2}\right)=\gamma-1$. The minimum value of $E$ (for $N=-1$ ) occurs at $v_{\perp}=0$, when $v_{\|}=\left(v_{\|}\right)_{m}$, the maximum value of $v_{\|}$. Specifically, we find that the minimum energy of an electron (in units of $m_{e} c^{2}$ ) for gyroresonant interaction with EMIC waves is

$$
E_{\min }=\gamma_{\min }-1=\left[1-\left(v_{\|}\right)_{m}^{2} / c^{2}\right]^{-1 / 2}-1,
$$

where

$$
\frac{\left(v_{\|}\right)_{m}}{c}=\frac{u\left[\epsilon^{2} x^{2}+\left\{\epsilon^{2} x^{2}+u^{2}\left(1-\epsilon^{2} x^{2}\right)\right\}^{1 / 2}\right]}{\epsilon^{2} x^{2}+u^{2}} .
$$

In equation (7), $x=\omega / \Omega_{H^{+}}$, as defined above, and the phase speed $u$ is given by the dispersion equation (2) or (4), as desired. Expressed in terms of practical units, result (6) gives the minimum resonant energy of an electron as 0.512 $E_{\min }(\mathrm{MeV})$, approximately.

[10] In Figure 3, the minimum resonant energy curves (6) for a plasma with $\eta_{1}=0.75, \eta_{2}=0.2, \eta_{3}=0.05$, are shown for the two cases $\alpha^{*}=10^{-3}, 10^{-2}$. The curves in Figure 3, which may be compared with the corresponding dispersion curves in Figure 2, demonstrate that (like the phase speed $u$ ) the minimum resonant energy $E_{\min }$ decreases as the parameter $\alpha^{*}$ decreases. Clearly, the electron minimum resonant energies are critically dependent on the wave frequency and on the local values of the electron number density $N_{0}$ and background magnetic field $B_{0}$ via the parameter $\alpha^{*}$. The
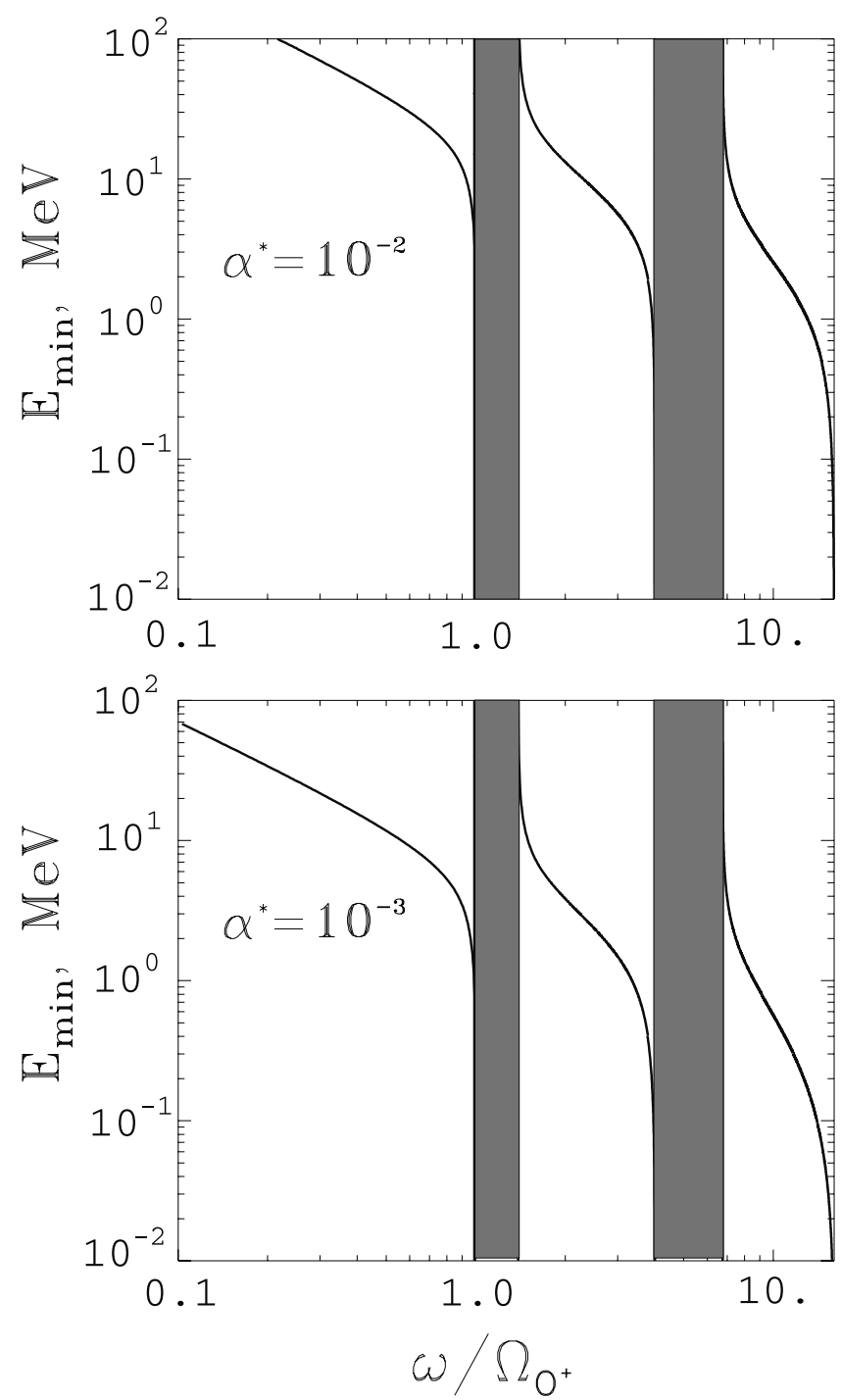

Figure 3. Profiles of minimum kinetic energy $E_{\min }$, given by equation (6) (using equations (7) and (2)), that an electron must possess for gyroresonant interaction with EMIC waves. The ion number densities are the same as in Figure 2. 

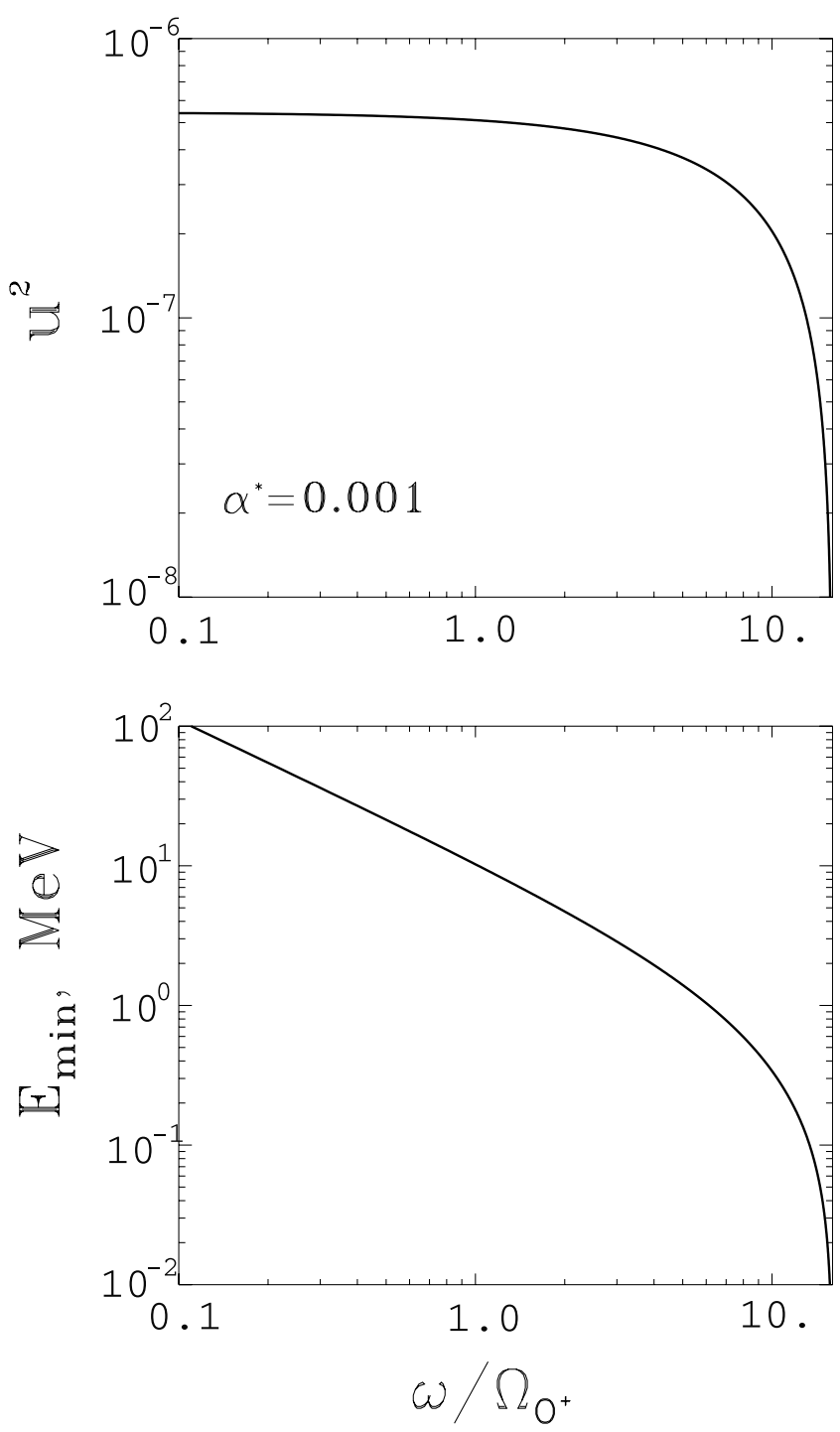

Figure 4. The upper curve is the dispersion curve, given by equation (4), for EMIC waves in an electron-proton plasma; the lower curve is the corresponding profile of the minimum kinetic energy of an electron for gyroresonance with EMIC waves, given by equation (6) (using equations (7) and (4)).

minimum resonant energy curve for an electron-proton plasma, given by equations (6), (7), and (4), is shown in the lower panel of Figure 4 for the case $\alpha^{*}=10^{-3}$; the upper panel shows the corresponding dispersion curve (4). Compared with an electron-proton plasma, the presence of heavy ions leads to lower resonant electron energies in the oxygen band and higher energies in the hydrogen band. In the helium band, resonant electron energies may be lower or higher according to the corresponding changes in the wave phase velocity (compare the upper panels of Figures 2 and $4)$. We defer consideration of the variation of $E_{\min }$ with the ion composition $\left(\eta_{1}, \eta_{2}, \eta_{3}\right)$ until section 5 .

\section{Pitch-Angle Diffusion Rates}

[11] The scattering of charged particles by small-amplitude, broadband electromagnetic waves may be treated by quasi-linear diffusion theory, e.g., see Kennel and Engelmann [1966], Melrose [1980], and Steinacker and Miller [1992]. Description of such wave-particle interactions involves solution of a kinetic equation for the particle distribution which contains diffusion coefficients due to cyclotron-resonant interactions of the particles with a prescribed spectrum of waves. Determination of expressions for the diffusion coefficients is straightforward, but their numerical evaluation can be involved, if multiple wave resonances need to be taken into account. Jordanova et al. [1996] calculated quasi-linear (pitch-angle, energy, mixed) diffusion coefficients for particles resonating with EMIC waves in a multi-ion plasma and applied the results to ion precipitation losses from the ring current during storms. In this paper we use a simple functional form for the pitch-angle diffusion coefficient $D_{\alpha \alpha}$ that can be deduced either from elementary arguments concerning the change in pitch-angle $\alpha$ of a particle over its gyroperiod $\tau_{g}$ due to resonant scattering waves (using $D_{\alpha \alpha} \approx$ $\left.(\Delta \alpha)^{2} / \tau_{g}\right)$, or by simplification of explicit expressions from formal quasi-linear theory given by Melrose [1980] and Steinacker and Miller [1992]. We assume wave propagation parallel to a uniform background magnetic field and obtain the expression

$$
D_{\alpha \alpha} \approx \frac{\left|\Omega_{e}\right|}{\gamma} \frac{k W(k)}{W_{0}}
$$

where $k$ is the resonant wavenumber; $W(k)$ is the wave magnetic field spectral density defined by

$$
W_{t o t}=\int_{k_{1}}^{k_{2}} W(k) d k
$$

where $W_{t o t}=(\Delta B)^{2} / 8 \pi$ is the total wave magnetic energy density; $W_{0}=B_{0}^{2} / 8 \pi$ is the background magnetic field energy density; and $\gamma$ is the Lorentz factor. The resonant wavenumber $k$ in (8) is given by

$$
\frac{c k}{\left|\Omega_{e}\right|}=\frac{1}{p|\cos \alpha|}
$$

where $p=\gamma v / c$ is the normalized electron momentum and $\alpha=\tan ^{-1}\left(v_{\perp} / v_{\|}\right)$is the electron pitch-angle. Relation (10) is a reexpression of the gyroresonance condition (5) with the $\omega$-term omitted, and $|N|=1$. Omission of the $\omega$-term is justified if $\omega /\left|\Omega_{e}\right| \ll 1$, a condition which is well satisfied for EMIC waves since for these waves $\omega / \Omega_{H^{+}}<1$. We note that result (8) for the pitch-angle diffusion coefficent of $D_{\alpha \alpha}$ is generic in the sense that it is valid, subject to a nondimensional factor of order unity on the right-hand side, for parallel-propagating small-amplitude $(\mathrm{R}$ mode or $\mathrm{L}$ mode) electromagnetic waves of general spectral density $\mathrm{W}$. It is convenient to express the reduced gyroresonance condition (10) in the form

$$
E(E+2) \cos ^{2} \alpha=\frac{\Omega_{e}^{2}}{c^{2} k^{2}}
$$

for which we have used the relation $p^{2}=E(E+2)$. Since we are considering a waveband with wavenumbers in the range 
$k_{1} \leq k \leq k_{2}$, it follows from equation (11) that electrons in resonance with the waves have kinetic energy $E$ and pitchangle $\alpha$ restricted by the condition

$$
\frac{\Omega_{e}^{2}}{c^{2} k_{2}^{2}} \leq E(E+2) \cos ^{2} \alpha \leq \frac{\Omega_{e}^{2}}{c^{2} k_{1}^{2}} .
$$

Therefore defining

$$
E_{1}=\left(1+\frac{\Omega_{e}^{2}}{c^{2} k_{1}^{2}}\right)^{1 / 2}-1, E_{2}=\left(1+\frac{\Omega_{e}^{2}}{c^{2} k_{2}^{2}}\right)^{1 / 2}-1
$$

and

$$
\begin{aligned}
& \alpha_{1}=\cos ^{-1}\left(\frac{\left|\Omega_{e}\right|}{c k_{1}} \cdot \frac{1}{[E(E+2)]^{1 / 2}}\right), \\
& \alpha_{2}=\cos ^{-1}\left(\frac{\left|\Omega_{e}\right|}{c k_{2}} \cdot \frac{1}{[E(E+2)]^{1 / 2}}\right)
\end{aligned}
$$

we deduce from equation (12) the following results: (1) If $E<E_{2}$, then no wave-particle resonance is possible. (2) If $E_{2}<E<E_{1}$, then resonance takes place over pitch-angles in the range $0 \leq \alpha \leq \alpha_{2}$. (3) If $E>E_{1}$, then resonance takes place over pitch-angles in the range $\alpha_{1} \leq \alpha \leq \alpha_{2}$. These results are useful in the construction of the curves for the pitch-angle diffusion coefficients below. The minimum resonant energy $E_{2}$ is an approximation to the value $E_{\text {min }}$ (defined by (6)), when the full resonance condition (5) is replaced by the reduced resonance condition (10). In the case of EMIC waves, $E_{2}$ is a very good approximation to $E_{\text {min }}$.

[12] It is convenient to transform from wavenumbers over the range $k_{1} \leq k \leq k_{2}$ to wave frequencies over the range $\omega_{1} \leq$ $\omega \leq \omega_{2}$. Writing

$$
\int_{k_{1}}^{k_{2}} W(k) d k=\int_{\omega_{1}}^{\omega_{2}} W(k(\omega)) \frac{d k}{d \omega} \cdot d \omega=\int_{\omega_{1}}^{\omega_{2}} \tilde{W}(\omega) d \omega
$$

where $k_{1}=k\left(\omega_{1}\right)$ and $k_{2}=k\left(\omega_{2}\right)$, we find that equation (8) can be written

$$
D_{\alpha \alpha} \approx \frac{\left|\Omega_{e}\right|}{\gamma W_{0}} \cdot \frac{2 k^{2} \tilde{W}}{(\omega)} \frac{d}{d w}\left(k^{2}\right)
$$

where, in equation (16), $k^{2}$ is a specified function of $\omega$ obtained from the wave dispersion relation, and $\tilde{W}$ is the wave frequency spectrum.

[13] A Gaussian frequency spectrum is assumed, namely,

$$
\tilde{W}(\omega) \propto \exp \left\{-\left[\left(\omega-\omega_{m}\right) / \delta \omega\right]^{2}\right\}
$$

where $\omega_{m}$ is the center frequency, with $\omega_{1}=\omega_{m}-\delta \omega$ and $\omega_{2}=\omega_{m}+\delta \omega$. Then equation (16) becomes

$$
D_{\alpha \alpha} \approx \frac{\left|\Omega_{e}\right|}{\gamma} \cdot \frac{R}{\nu \delta \omega} \cdot \frac{2 k^{2}}{\frac{d}{d w}\left(k^{2}\right)} e^{-\left[\left(\omega-\omega_{m}\right) / \delta \omega\right]^{2}},
$$

where

$$
R=\frac{W_{t o t}}{W_{0}}=\frac{(\triangle B)^{2}}{B_{0}^{2}}
$$

is the ratio of the turbulent wave energy to the background magnetic field energy; the parameter $\nu$, which is given by $v=\sqrt{\pi} \operatorname{erf}(1)(\approx 1.49)$, where erf is the error function, arises from the normalization of $\tilde{W}$.

[14] In order to calculate $D_{\alpha \alpha}$ for EMIC waves in a multiion plasma, we evaluate equation (18) using the dispersion relation (2) to obtain

$$
D_{\alpha \alpha} \approx \frac{\left|\Omega_{e}\right|}{(E+1)} \cdot \frac{2 R}{\nu \delta x} \cdot\left(\frac{\alpha^{*} \epsilon x^{2}-Y}{2 \alpha^{*} \epsilon x-Z}\right) e^{-\left[\left(x-x_{m}\right) / \delta x\right]^{2}},
$$

where

$$
\begin{aligned}
Y & =x\left\{\frac{1}{1+\epsilon x}+\frac{\eta_{1}}{x-1}+\frac{\eta_{2}}{4 x-1}+\frac{\eta_{3}}{16 x-1}\right\}, \\
Z & =\frac{1}{(1+\epsilon x)^{2}}-\frac{\eta_{1}}{(x-1)^{2}}-\frac{\eta_{2}}{(4 x-1)^{2}}-\frac{\eta_{3}}{(16 x-1)^{2}}, \\
x_{m} & =\omega_{m} / \Omega_{H^{+}}, \delta x=\delta \omega / \Omega_{H^{+}},
\end{aligned}
$$

and, as above, $x=\omega / \Omega_{H^{+}}$. Associated with formula (20) is the expression for the resonant pitch-angle, obtained from equation (11), which is

$$
\cos \alpha=\left[E(E+2)\left(\epsilon^{2} x^{2}-\frac{\epsilon}{\alpha^{*}} Y\right)\right]^{-1 / 2} .
$$

[15] To plot $D_{\alpha \alpha}$ as a function of $\alpha$, for a given value of $E$, we use the above results concerning the pitch-angles over which resonance takes place, which involve the energies $E_{1}$ and $E_{2}$ defined by equation (13) and proceed as follows. First, we set values for the basic input parameters, i.e., $\left|\Omega_{e}\right|$, $R, \alpha^{*}, \eta_{1}, \eta_{2}, \eta_{3}, \omega_{1}$, and $\omega_{2}$; note that the parameters $\omega_{m}=$ $\left(\omega_{1}+\omega_{2}\right) / 2$ and $\delta \omega=\left(\omega_{2}-\omega_{1}\right) / 2$ are thereby specified. We then calculate $E_{1}$ and $E_{2}$ from equations (13) and (1). For $E>E_{1}$, we choose a desired value of $E$, and set $\omega=\omega_{2}$ (or, equivalently, $x=\omega_{2} / \Omega_{H^{+}}$) in equations (22) and (20). This yields the value of $D_{\alpha \alpha}$ at the pitch-angle $\alpha_{2}$ (defined by equation (14)). In accord with result (3) (following equation (14)), we then calculate $\alpha$ and $D_{\alpha \alpha}$ from equations (22) and (20) for a chosen set of decreasing frequencies as far as the frequency $\omega_{1}$, which corresponds to the pitch-angle $\alpha_{1}$ (also defined by equation (14)). Thus a plot of $D_{\alpha \alpha}$ against $\alpha$ is constructed by treating equations (20) and (22) as parametric equations with parametric variable $x$. For $E_{2}<E<$ $E_{1}$, the plotting procedure is similar, but must be modified, in accord with result (2). In this case, for a chosen value of $E$ the plotting is initiated at $\omega=\omega_{2}$, corresponding to $\alpha_{2}$, proceeds with a chosen set of decreasing frequencies, but terminates when $\alpha=0$, which corresponds to the frequency, say $\omega_{3}\left(>\omega_{1}\right)$, at which the right-hand side of equation (22) is unity.

[16] For the simpler case of EMIC waves in an electronproton plasma, we evaluate the cyclotron-resonant pitchangle diffusion coefficient $D_{\alpha \alpha}$ given by equation (18) 


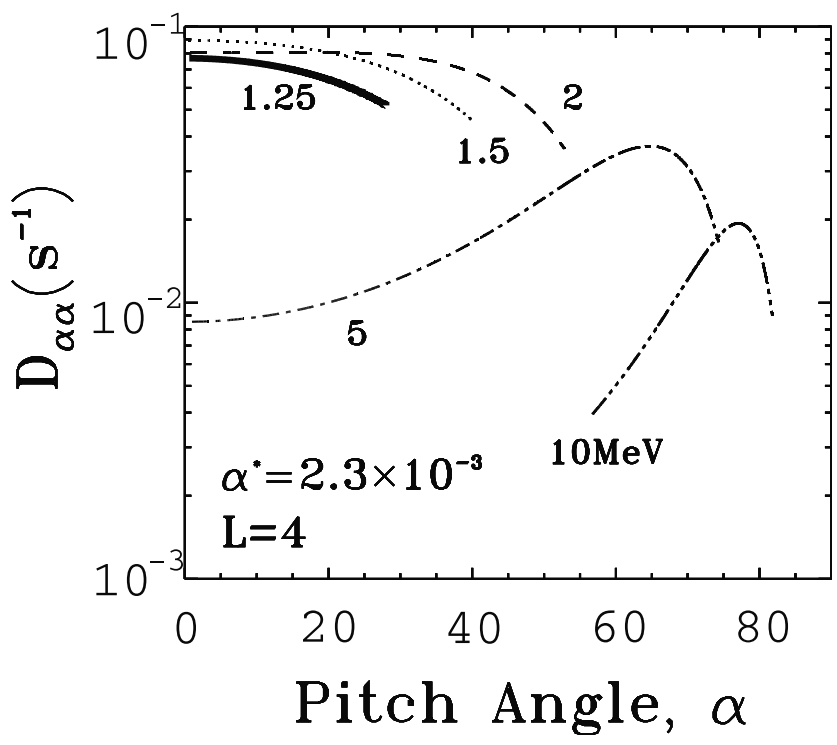

Figure 5. Pitch-angle diffusion coefficient $D_{\alpha \alpha}$, given by equation (24), corresponding to EMIC waves in an electronproton plasma with amplitude $\Delta B=1 n T$ and Gaussian frequency spectrum with center $\omega_{m}=\Omega_{H^{+}} / 3$ and semi-bandwidth $\delta \omega=\Omega_{H^{+}} / 6 ; L=4$.

using an approximated form of the dispersion relation (4), namely

$$
u^{2}=\alpha^{*} \epsilon(1-x)
$$

The result is

$$
D_{\alpha \alpha} \approx \frac{\left|\Omega_{e}\right|}{(E+1)} \cdot \frac{2 R}{\nu \delta x} \cdot \frac{x(1-x)}{(2-x)} \cdot e^{-\left[\left(x-x_{m}\right) / \delta x\right]^{2}}
$$

with

$$
\cos \alpha=\frac{1}{x}\left[\frac{\alpha^{*}(1-x)}{\epsilon E(E+2)}\right]^{1 / 2},
$$

where all symbols are as previously defined. Once the basic input parameters, $\left|\Omega_{e}\right|, R, \alpha^{*}, \omega_{1}$, and $\omega_{2}$, are prescribed, construction of plots of $D_{\alpha \alpha}$ as a function of $\alpha$, from (24) and (25), proceeds as outlined above for a multi-ion plasma.

[17] Equation (20) provides a simple analytical expression for the scattering rate of relativistic electrons by EMIC waves in a multi-ion plasma environment. The analysis is strictly only valid for the case of first-order cyclotron resonance with field-aligned waves. For the general case of oblique wave propagation, higher-order scattering can also occur, but for moderate angles of propagation the firstorder resonance will dominate, especially at energies just above the minimum resonant value and for pitch-angles near the loss cone. A full quasi-linear analysis of the scattering rate of relativistic electrons was originally performed by Lyons and Thorne [1972] for the case of oblique EMIC waves in an electron-proton plasma. Figure 5 shows the local diffusion rate obtained from equation (24) using the equatorial plasma and wave parameters employed by
Lyons and Thorne [1972], namely $\alpha^{*}=2.3 \times 10^{-3}, \Delta B=$ $1 n T, \omega_{m}=\Omega_{H^{+}} / 3$, and $\delta \omega=\Omega_{H^{+}} / 6$. The results of Lyons and Thorne [1972] were averaged over the bounce orbit of the electrons, which resulted in an order-of-magnitude reduction in the bounce averaged rates of scattering compared to local equatorial values. However, apart from this important difference, the simple first-order scattering results obtained from equation (24) provide an excellent representation of the energy and pitch-angle dependence for the quasi-linear diffusion rates. We therefore feel confident in using our simple formula (20) to investigate the values of $D_{\alpha \alpha}(E, \alpha)$ in a multi-ion plasma.

[18] It is useful to compare the magnitudes of the pitchangle diffusion coefficients $D_{\alpha \alpha}$ for electrons scattered by EMIC waves with the "strong" pitch-angle diffusion rate as defined by Kennel [1969]. The mean lifetime or precipitation time $\tau_{S D}$ of particles under "strong" pitch-angle diffusion has been calculated by Lyons [1973] and further refined by Schulz [1974]. For a small equatorial loss cone of half-angle $\alpha_{0}$, the result can be written

$$
\tau_{S D} \approx \frac{64 L R_{E}}{35(1-A) v \alpha_{0}^{2}}
$$

where $v$ is the particle speed, $L$ is the magnetic shell parameter, $R_{E}$ is the radius of the Earth, and $A$ is the particle albedo from the atmosphere at either foot of the magnetic field line. The condition for strong diffusion is that $D_{0} \geq$ $\alpha_{0}^{2} / \tau_{B}$, where $D_{0}$ is the bounce-averaged value of the pitchangle diffusion coefficient $D_{\alpha \alpha}$, at $\alpha=\alpha_{0}$, and $\tau_{B}$ is the quarter-bounce time for particles with zero pitch-angle. Thus under strong diffusion, particles diffuse across the loss cone in less than a quarter-bounce period, and the particle flux within the loss cone approaches isotropy. Moreover, the particle precipitation rate is then insensitive to the magnitude of the diffusion coefficient. For a dipole magnetic field the loss cone size at $L R_{E}$ is given approximately by $\alpha_{0}=L^{-3 / 2}(4-3 / L)^{-1 / 4}$. Taking $A=0.25$, and defining the rate of strong pitch-angle diffusion as $D_{S D}=1 / \tau_{S D}$, we obtain from equation (26) the expression

$$
D_{S D} \approx \frac{9 \cdot 66}{L^{4}}\left[\frac{4 L}{4 L-3}\right]^{1 / 2} \frac{[E(E+2)]^{1 / 2}}{(E+1)}\left(s^{-1}\right) .
$$

In Figure 6 we show plots of $D_{S D}$ for electrons of a specified energy $E$, as a function of $L . D_{S D}$ is a monotonically decreasing function of $L$ and is essentially independent of $E$ for energies exceeding $1 \mathrm{MeV}$. We compare our results for $D_{\alpha \alpha}$ with corresponding values of $D_{S D}$ in the following section.

\section{Results and Discussion}

[19] The densities of the major ion species, $\mathrm{H}^{+}, \mathrm{He}^{+}$, and $\mathrm{O}^{+}$, in the inner magnetosphere $(L<7)$ are dependent on magnetic local time (MLT), L-value, latitude, and geomagnetic and solar activity [Young et al., 1977, 1982; Craven et al., 1997]. The helium $\left(\mathrm{He}^{+}\right)$composition is solar cyclic and MLT dependent, while the oxygen $\left(\mathrm{O}^{+}\right)$composition is solar cyclic and geomagnetic activity dependent. The fractional helium density $\eta_{2}$ typically varies from 0.02 to 0.1 during solar minimum, from 0.05 to 0.25 during solar 




Figure 6. Strong pitch-angle diffusion coefficient $D_{S D}$, given by equation (27), as a function of $L$, for the specified values of the electron kinetic energy $E$.

maximum, and can reach 0.3 as an extreme value. The fractional oxygen density $\eta_{3}$ typically varies from 0.01 to 0.1 over the solar cycle and reaches extreme values near 0.25 during intense magnetic storms. The fractional ion densities $\eta_{1}, \eta_{2}$, and $\eta_{3}$ affect the EMIC wave phase speed through the dispersion relation (2) and hence affect the electron minimum resonant energy $E_{\min }$ given by equation (6). $E_{\min }$ also decreases as the wave frequency increases towards the gyrofrequency in each wave band (Figure 3).

[20] In Figure 7 we show the results of a comprehensive examination of the variation of $E_{\min }$ with $\eta_{1}, \eta_{2}$, and $\eta_{3}$ for reasonable (but optimum) values of the wave frequency in each propagation band. The figure also shows the dependence of $E_{\min }$ on the cold-plasma parameter $\alpha^{*}$ given by equation (3). The upper, middle, and lower panels of Figure 7 refer to the hydrogen $\left(\mathrm{H}^{+}\right)$, helium $\left(\mathrm{He}^{+}\right)$, and oxygen $\left(\mathrm{O}^{+}\right)$ bands, respectively. For each band, we find that $E_{\text {min }}$ depends significantly on the fractional density of the ion that specifies the band but is weakly dependent on the other two fractional ion densities (e.g., for the $\mathrm{H}^{+}$band, $E_{\text {min }}$ varies significantly with $\eta_{1}$, but is weakly dependent on $\eta_{2}$ and $\eta_{3}$ ). Therefore we show the variation of $E_{\min }$ with the respective fractional densities $\eta_{1}, \eta_{2}$, and $\eta_{3}$ in the upper, middle, and lower panels of Figure 7 . For the $\mathrm{H}^{+}$band, we set $\omega / \Omega_{O^{+}}=11, \eta_{2}=2 \eta_{3}$, and let $\eta_{1}$ vary from 0.7 to 1 . Thus in this case, $0 \leq \eta_{2} \leq 0.2$ and $0 \leq \eta_{3} \leq 0.1$. For the $\mathrm{He}^{+}$ band, we set $\omega / \Omega_{\hat{O}^{+}}=3.5$, and specify the ion densities by $0.65 \leq \eta_{1} \leq 0.95,0 \leq \eta_{2} \leq 0.3$, and $\eta_{3}=0.05$. For the $\mathrm{O}^{+}$ band we set $\omega / \Omega_{O^{+}}=0.95$ and specify the ion densities by $0.65 \leq \eta_{1} \leq 0.9, \eta_{2}=0.1$, and $0 \leq \eta_{3} \leq 0.25$. Notably, $E_{\text {min }}$ decreases as the fractional composition increases for the ion that specifies the band, and for specified values of the ion densities and frequency $\omega, E_{\text {min }}$ decreases as $\alpha^{*}$ decreases. For each panel of Figure 7 the region lying between the profiles corresponding to $\alpha^{*}=10^{-3}$ and $\alpha^{*}=10^{-2}$ specifies the typical range of minimum kinetic energies for electrons in resonance with EMIC waves inside the plasmapause, for the given values of wave frequency and ion composition. Since the absolute value of $E_{\min }$ depends
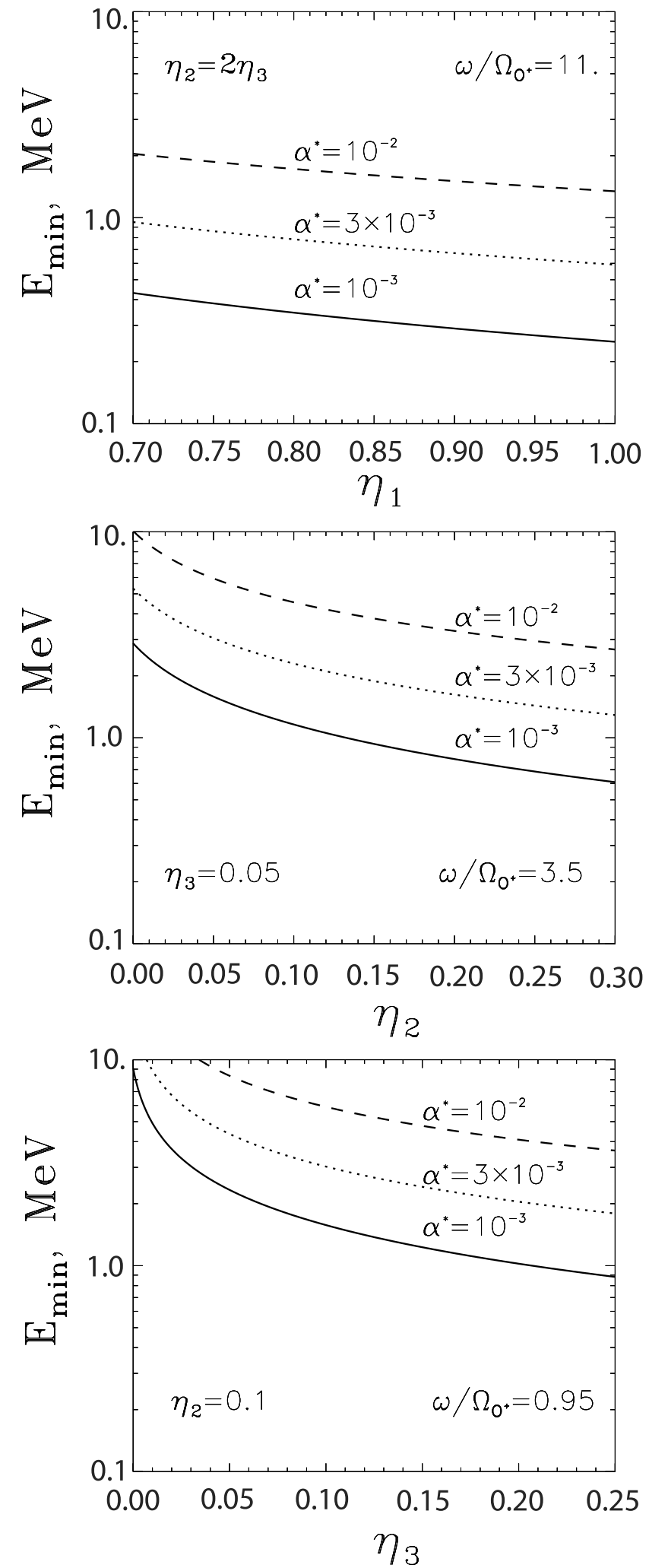

Figure 7. Minimum electron kinetic energy curves for gyroresonance with EMIC waves, given by equation (6), as functions of the ion composition, for the specified values of the wave frequency and parameter $\alpha^{*} ; \eta_{1}, \eta_{2}$, and $\eta_{3}$ are the hydrogen $\left(\mathrm{H}^{+}\right)$, helium $\left(\mathrm{He}^{+}\right)$, and oxygen $\left(\mathrm{O}^{+}\right)$fractional ion number densities respectively, with $\eta_{1}+\eta_{2}+\eta_{3}=1$. The specified frequencies in the upper, middle, and lower panels typify the hydrogen, helium, and oxygen bands, respectively. 

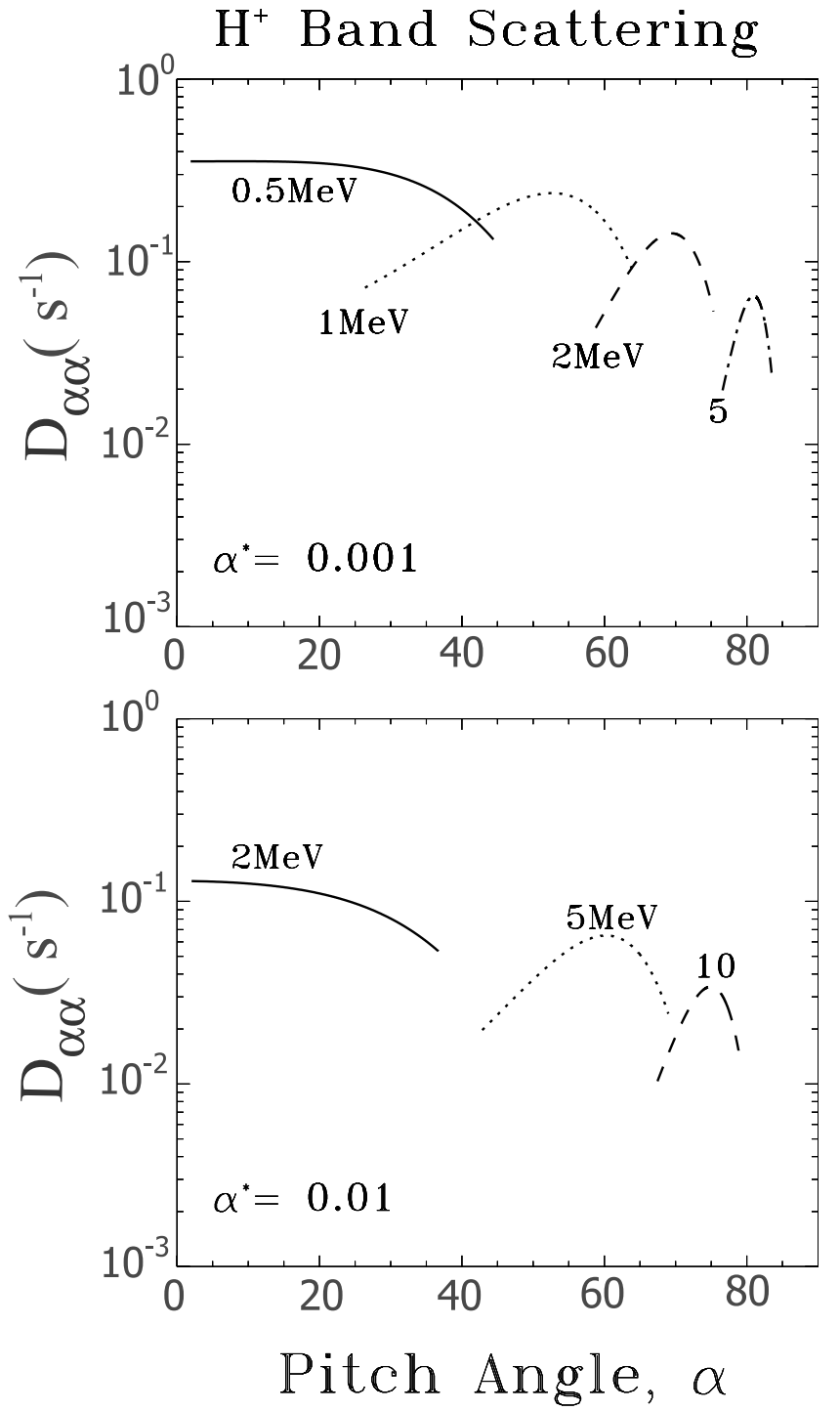

Figure 8. Pitch-angle diffusion coefficient $D_{\alpha \alpha}$, given by equation (20), corresponding to hydrogen band EMIC waves in a multi-ion plasma with amplitude $\Delta B=1 n T$, and Gaussian frequency spectrum with center $\omega_{m}=0.6 \Omega_{H^{+}}$and semi-band-width $\delta \omega=0.1 \Omega_{H^{+}} ; L=4$ and $\eta_{1}=0.85, \eta_{2}=$ $0.1, \eta_{3}=0.05$.

sensitively on the adopted wave frequencies (Figure 3), one should not draw definitive conclusions on the relative importance of resonant scattering in the three propagation bands until better observational information of the wave spectral properties is available.

[21] Local rates of the pitch-angle diffusion $D_{\alpha \alpha}(E, \alpha)$, calculated from equation (20), are shown in Figures 8, 9, and 10 , respectively, for $\mathrm{H}^{+}$band, $\mathrm{He}^{+}$band, and $\mathrm{O}^{+}$band waves, in each case for the wave amplitudes $\Delta B=\ln T$, and location $L=4$. The parameters adopted for the $\mathrm{H}^{+}$band waves $\left(\eta_{1}=0.85, \eta_{2}=0.1, \eta_{3}=0.05, \omega_{m}=0.6 \Omega_{H^{+}}\right.$, and $\left.\delta \omega=0.1 \Omega_{H^{+}}\right)$are intended to characterize EMIC waves excited during the sudden commencement phase of a magnetic storm [e.g., Bräysy et al., 1998]. For values of $\alpha^{*}$ between $10^{-3}$ (Figure 8, upper panel) and $10^{-2}$ (Figure 8 , lower panel) the minimum energy $E_{2}$ varies between 292
$\mathrm{keV}$ and $1.52 \mathrm{MeV}$. The "transition" energy $E_{1}$ varies between $860 \mathrm{keV}$ and $3.55 \mathrm{MeV}$. The scattering rates shown in Figure 8 represent optimum conditions for resonance at the equator for each specified value of $\alpha^{*}$. For resonant energies just above the minimum value $E_{2}$ the scattering rates maximize near the loss cone, while higher energy electrons are scattered more effectively at larger pitch-angle. As electrons execute their bounce $\left(\tau_{B} \leq 1 s\right)$ and azimuthal gradient drift $\left(\tau_{D} \leq 10 \mathrm{~min}\right)$ motions, the conditions for gyroresonance with EMIC waves change. Rates of EMIC wave scattering, over time-scales larger than the drift time $\tau_{D}$, should be averaged over both the electron bounce and drift orbits. Resonant electron energies increase at higher latitudes and the bounce-averaged scattering rates [e.g., Lyons and Thorne, 1972] near the loss cone are typically an order of magnitude lower than the local equatorial values shown in Figure 8. Nonetheless, for typical EMIC wave
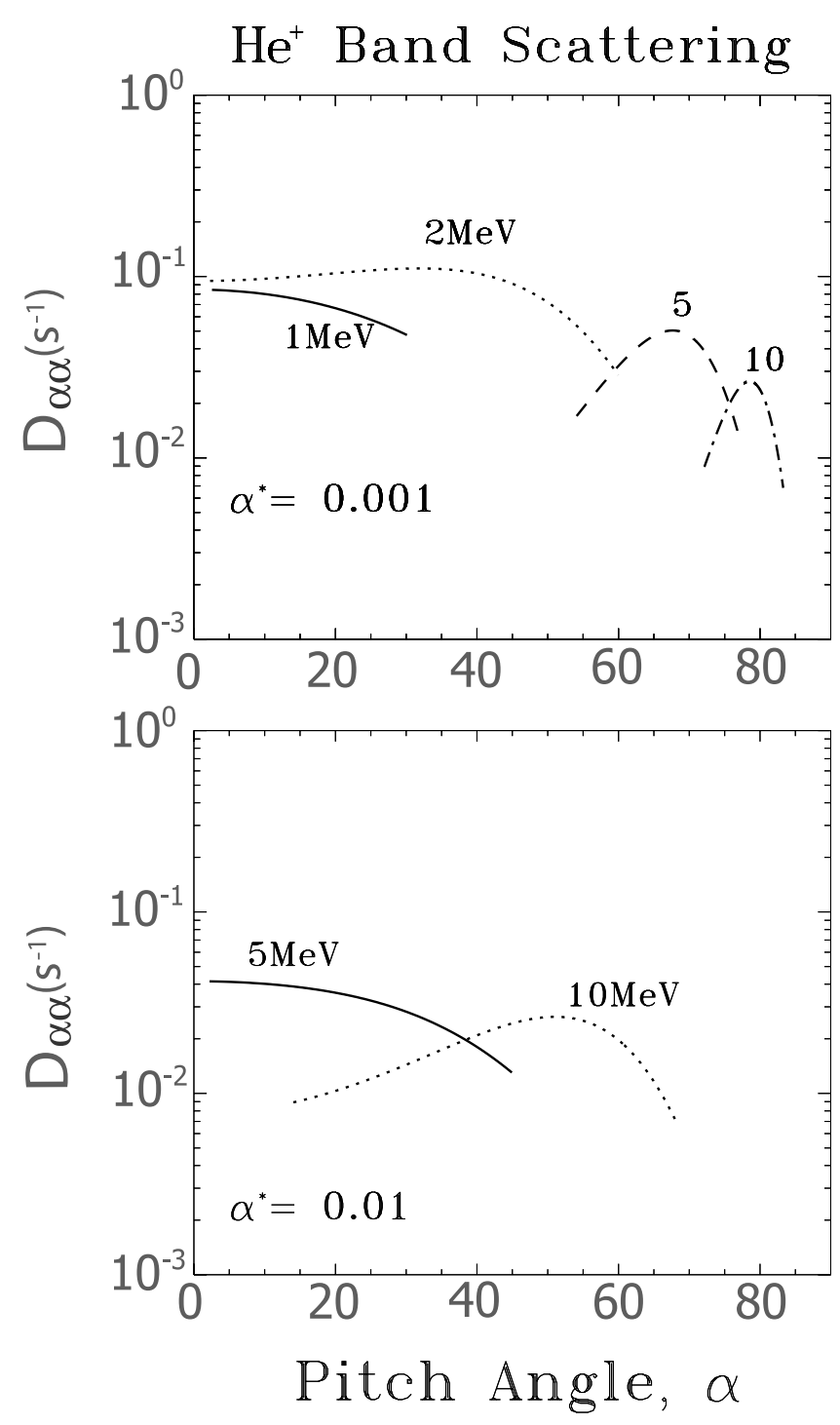

Figure 9. Pitch-angle diffusion coefficient $D_{\alpha \alpha}$, given by equation (20), corresponding to helium band EMIC waves in a multi-ion plasma with amplitude $\Delta B=1 n T$ and Gaussian frequency spectrum with center $\omega_{m}=3 \Omega_{O^{+}}$and semi-bandwidth $\delta \omega=0.5 \Omega_{O^{+}} ; L=4$ and $\eta_{1}=0.7, \eta_{2}=0.2, \eta_{3}=0.1$. 

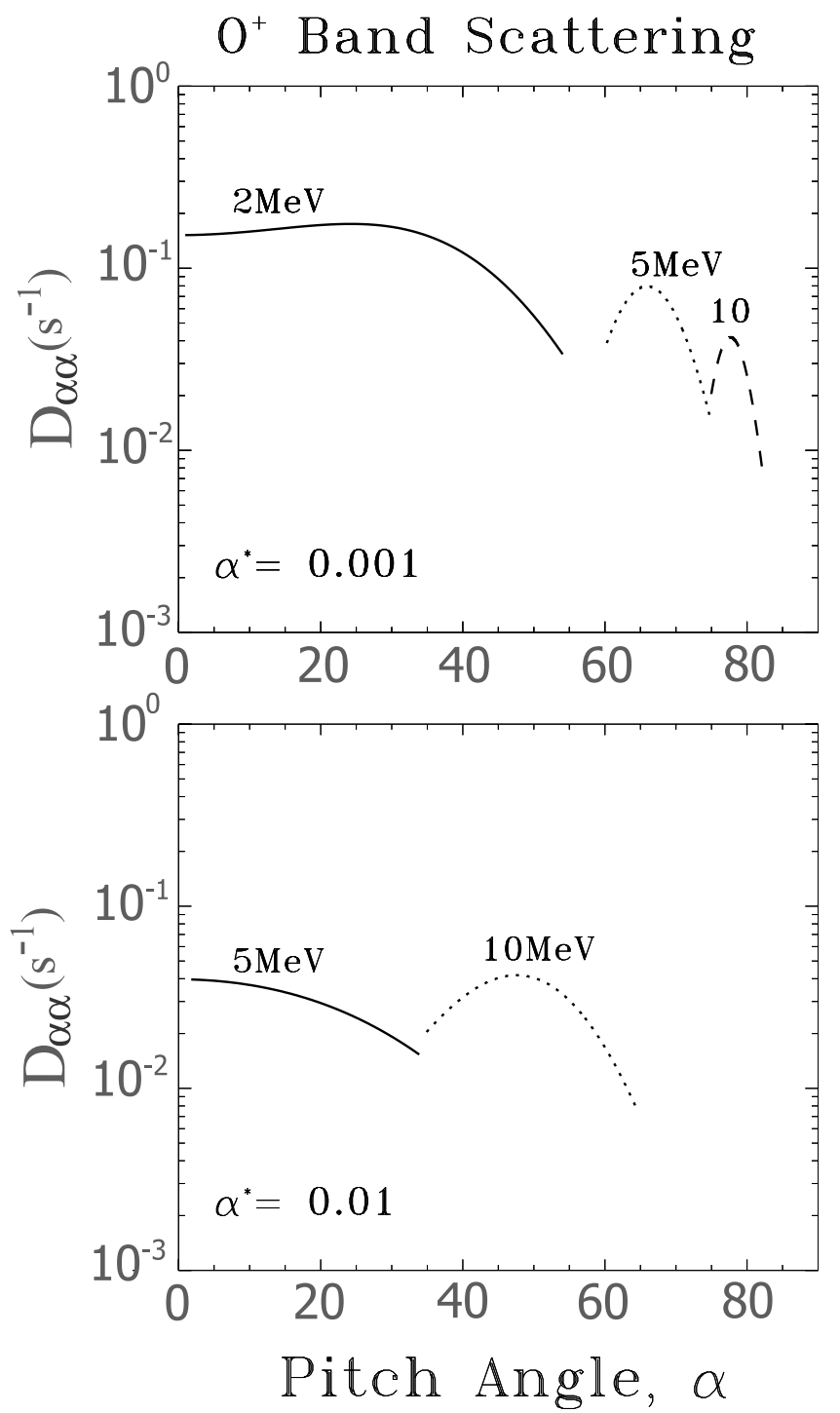

Figure 10. Pitch-angle diffusion coefficient $D_{\alpha \alpha}$, given by equation (20), corresponding to oxygen band EMIC waves in a multi-ion plasma with amplitude $\Delta B=1 n T$ and Gaussian frequency spectrum with center $\omega_{m}=0.9 \Omega_{O^{+}}$and semi-band-width $\delta \omega=0.05 \Omega_{O^{+}} ; L=4$ and $\eta_{1}=0.6, \eta_{2}=$ $0.2, \eta_{3}=0.2$.

amplitudes $(\Delta B>1 n T)$ observed near the duskside plasmapause [Fraser and Nguyen, 2001], bounce-averaged scattering rates near the loss cone should exceed the strong diffusion level (Figure 6). Such rapid scattering will lead to intense relativistic electron $(\geq 1 \mathrm{MeV})$ precipitation in localized regions where the waves are present. The recent X-ray observations of Millan [2002] and theoretical models for the simulation of EMIC wave excitation during storm conditions [Jordanova et al., 1998, 2001] suggest that the region of rapid scattering is extremely localized and might typically occur over $1 \%$ of the electron drift orbit. The driftaveraged lifetime for trapped electron decay due to EMIC scattering during the onset of a storm will therefore be $\tau_{L} \approx$ $100 \tau_{S D}$ or several hours, which is short enough to account for the rapid removal of the relativistic electron population in the outer radiation zone during the onset of a storm.
Rapid removal of electrons during the main phase of a storm has hitherto been attributed to the so-called Dst effect [e.g., Kim and Chan, 1997; see also Li et al., 1997]. The calculations reported in the present paper therefore show that relativistic electron pitch-angle scattering by EMIC waves can compete with the Dst effect as a mechanism for depleting relativistic electrons from the outer zone during the initial and main phases of a magnetic storm.

[22] Figure 9 shows local equatorial values of $D_{\alpha \alpha}(E, \alpha)$ corresponding to $\mathrm{He}^{+}$band waves for parameters that typify the main phase and recovery phase of a storm $\left(\eta_{1}=0.7, \eta_{2}=\right.$ $0.2, \eta_{3}=0.1, \omega_{m}=3 \Omega_{O^{+}}$, and $\delta \omega=0.5 \Omega_{O^{+}}$). The scattering rates in Figure 9 are slightly smaller than those in Figure 8, and the typical minimum resonant energies $E_{2}$ are higher, namely $819 \mathrm{keV}$ for $\alpha^{*}=10^{-3}$, and $3.4 \mathrm{MeV}$ for $\alpha^{*}=$ $10^{-2}$. The precise value of the resonant electron energies are dependent on the assumptions made for the wave spectral properties. Nevertheless, one may conclude that relativistic electrons ( $E$ greater than a few $\mathrm{MeV}$ ) can be removed by $\mathrm{He}^{+}$band scattering on a time-scale of several hours during the main phase and recovery phase of a storm. Such EMIC wave-induced precipitation could be responsible for the hard X-ray events observed by Millan [2002] in the recovery phase of a moderate storm.

[23] Scattering rates $D_{\alpha \alpha}(E, \alpha)$ for $\mathrm{O}^{+}$band waves are shown in Figure 10 for parameters representative of the main phase of a storm $\left(\eta_{1}=0.6, \eta_{2}=0.2, \eta_{3}=0.2, \omega_{m}=\right.$ $0.9 \Omega_{O^{+}}$, and $\left.\delta \omega=0.05 \Omega_{O^{+}}\right)$. Even for the extremely high normalized frequencies adopted for these calculations, the resonant electron energies in the $\mathrm{O}^{+}$band are higher than those in the $\mathrm{He}^{+}$band (see Figure 9). Therefore the $\mathrm{O}^{+}$band waves, whenever present, will probably only affect the extremely relativistic electron population.

[24] Several assumptions have been made in our analysis to obtain simple analytical formulae for the rates of electron scattering. The most severe is the assumption that the EMIC waves propagate strictly parallel to the background magnetic field, which allows us to ignore the effects of higher harmonic scattering. To justify this simplifying assumption, we refer to the study of Lyons and Thorne [1972] who found that the contribution from the first cyclotron harmonic resonant scattering dominates, even for moderately large angles of propagation. The expressions derived in the present paper for $D_{\alpha \alpha}(E, \alpha)$ are local values, and these should be averaged over both the bounce motion [e.g., Lyons and Thorne, 1972] and the drift path of resonant electrons to obtain realistic electron lifetimes. This requires detailed knowledge of the power spectral intensity of EMIC waves over the entire outer radiation zone throughout the different phases of a storm. Such wave information is currently unavailable, but will be required for a complete analysis of the effects of EMIC wave scattering on relativistic electron dynamics during storms.

\section{Concluding Remarks}

[25] The concept of stormtime strong diffusion scattering and rapid selected loss of relativistic electrons by EMIC waves was first discussed by Thorne and Kennel [1971]. Detailed quasi-linear calculations of the bounce-averaged diffusion rate in an electron-proton plasma [Lyons and Thorne, 1972] demonstrated that electrons with energy above 
$1 \mathrm{MeV}$ should be subject to strong diffusion scattering in regions where strong $(\Delta B \geq 1 n T)$ EMIC waves are present. Since those early studies, the importance of the heavy ion population on EMIC wave propagation and excitation has been firmly established, but until the present study no attempt has been made to apply electron-cyclotron resonant pitchangle diffusion rates for EMIC waves in a multi-ion plasma to relativistic electron precipitation during storms.

[26] Our new results confirm that stormtime EMIC waves can indeed cause strong diffusion precipitation of relativistic $(\leq 1 \mathrm{MeV})$ electrons under appropriate conditions. The predominant local condition required to lower electron resonant energies to geophysically interesting values is a suitably low value of the parameter $\alpha^{*}=\Omega_{e}^{2} / \omega_{p e}^{2}$. Typically, $\alpha^{*}=10^{-3}$ is sufficient to ensure minimum electron resonant energies of $\leq 1 \mathrm{MeV}$. Such conditions are only found in regions of high plasma density and low magnetic field, such as the duskside plasmasphere or within detached plasma regions at high L-values. A second important condition required to lower electron resonant energies close to $1 \mathrm{MeV}$ is the presence of EMIC waves at frequencies approaching the ion gyrofrequency in any of the propagation bands. A third condition to lower electron resonant energies in the $\mathrm{He}^{+}$and $\mathrm{O}^{+}$bands is enhanced concentrations of heavy ions which occur during solar maximum conditions and during geomagnetic storms. If one or more of these conditions hold, then relativistic electrons can be removed from the outer zone by EMIC wave scattering over a time-scale of several hours to a day.

[27] We have carried out detailed calculations of the pitchangle diffusion coefficient $D_{\alpha \alpha}$ for cyclotron resonant interaction between electrons and EMIC waves in a multi-ion $\left(\mathrm{H}^{+}, \mathrm{He}^{+}, \mathrm{O}^{+}\right)$plasma using plasma and wave parameters characteristic of storm conditions. We have demonstrated that the pitch-angle scattering by EMIC waves can provide an efficient mechanism for the loss of relativistic electrons from the Earth's outer radiation belt during different phases of a magnetic storm. Localized regions of intense relativistic electron precipitation should be observed predominantly in the dusk sector [e.g., Millan, 2002] where the EMIC wave scattering is most effective. Future detailed modeling of the power spectral density of EMIC waves as a function of location and geomagnetic activity, together with calculations of the bounce and drift averaged rates of scattering for all harmonic resonances, will be required to quantify fully the importance of such scattering on the loss of outer-zone relativistic electrons. Other wave modes can contribute to the scattering and their effects will also need to be included in future modeling of relativistic electron dynamics during storms.

[28] Acknowledgments. This work is supported in part by the Natural Sciences and Engineering Research Council of Canada under grant A-0621. Additional support is acknowledged from NSF grant ATM 97 29021 and NASA grant NAG5 11922. We thank Fuliang Xiao for assistance in the construction of the figures.

[29] Arthur Richmond thanks Vania K. Jordanova and Kirsten R. Lorentzen for their assistance in evaluating this paper.

\section{References}

Anderson, B. J., R. E. Erlandson, and L. J. Zanetti, A statistical study of Pc 1-2 magnetic pulsations in the equatorial magnetosphere, 1, Equatorial occurrence distributions, J. Geophys. Res., 97, 3075, 1992a.
Anderson, B. J., R. E. Erlandson, and L. J. Zanetti, A statistical study of Pc 1-2 magnetic pulsations in the equatorial magnetosphere, 2 , Wave properties, J. Geophys. Res., 97, 3089, 1992b.

Bräysy, T., K. Mursula, and G. Marklund, Ion cyclotron waves during a great magnetic storm observed by Freja double-probe electric field instrument, J. Geophys. Res., 103, 4145, 1998.

Callis, L. B., M. Natarajan, J. D. Lambeth, and D. N. Baker, Solar atmospheric coupling by electrons (SOLACE), 2, Calculated stratospheric effects of precipitating electrons, 1979-1988, J. Geophys. Res., 103, 28,421, 1998.

Cornwall, J. M., F. V. Coroniti, and R. M. Thorne, Turbulent loss of ring current protons, J. Geophys. Res., 75, 4699, 1970.

Craven, P. D., D. L. Gallagher, and R. H. Comfort, Relative concentration of $H_{e}^{+}$in the inner magnetosphere as observed by the DE 1 retarding ion mass spectrometer, J. Geophys. Res., 102, 2279, 1997.

Elkington, S. R., M. K. Hudson, and A. A. Chan, Acceleration of relativistic electrons via drift-resonant interaction with toroidal-mode Pc-5 ULF oscillations, Geophys. Res. Lett., 26, 3273, 1999.

Erlandson, R. E., and A. J. Ukhorskiy, Observations of electromagnetic ion cyclotron waves during geomagnetic storms: Wave occurrence and pitch angle scattering, J. Geophys. Res., 106, 3883, 2001.

Foat, J. E., R. P. Lin, D. M. Smith, F. Fenrich, R. Millan, I. Roth, K. R. Lorentzen, M. P. McCarthy, G. K. Parks, and J. P. Treilhou, First detection of a terrestrial MeV X-ray burst, Geophys. Res. Lett., 25, 4109, 1998.

Fraser, B. J., and T. S. Nguyen, Is the plasmapause a preferred source region of electromagnetic ion cyclotron waves in the magnetosphere?, J. Atmos. Sol.-Terr. Phys., 63, 1225, 2001.

Fraser, B. J., J. C. Samson, Y. D. Hu, R. L. McPherron, and C. T. Russell, Electromagnetic ion cyclotron waves observed near the oxygen cyclotron frequency by ISEE 1 and 2, J. Geophys. Res., 97, 3063, 1992.

Fraser, B. J., H. J. Singer, W. J. Hughes, J. R. Wygant, R. R. Anderson, and Y. D. Hu, CRRES Poynting vector observations of electromagnetic ion cyclotron waves near the plasmapause, J. Geophys. Res., 101, 15,331, 1996.

Friedel, R. H. W., G. D. Reeves, and T. Obara, Relativistic electron dynamics in the inner magnetosphere-A review, J. Atmos. Sol.-Terr. Phys., $64,265,2002$

Gallagher, D. L., P. D. Craven, and R. H. Comfort, Global core plasma model, J. Geophys. Res., 105, 18,819, 2000.

Horne, R. B., and R. M. Thorne, On the preferred source location for the convective amplification of ion cyclotron waves, J. Geophys. Res., 98, 9233, 1993

Horne, R. B., and R. M. Thorne, Convective instabilities of electromagnetic ion cyclotron waves in the outer magnetosphere, J. Geophys. Res., 99, $17,259,1994$

Horne, R. B., and R. M. Thorne, Potential waves for relativistic electron scattering and stochastic acceleration during magnetic storms, Geophys. Res. Lett., 25, 3011, 1998.

Hudson, M. K., S. R. Elkington, J. G. Lyon, M. J. Wiltberger, and M. Lessard, Radiation belt electron acceleration by ULF wave drift resonance: Simulation of 1997 and 1998 storms, in Space Weather, Geophys. Monogr. Ser., vol. 125, edited by P. Song, H. J. Singer, and G. L. Siscoe, p. 289, AGU, Washington, D. C., 2001.

Jordanova, V. K., J. U. Kozyra, and A. F. Nagy, Effects of heavy ions on the quasi-linear diffusion coefficients from resonant interactions with electromagnetic ion cyclotron waves, J. Geophys. Res., 101, 19,771, 1996.

Jordanova, V. K., C. J. Farrugia, J. M. Quinn, R. M. Thorne, K. W. Ogilvie, R. P. Lepping, G. Lu, A. J. Lazarus, M. F. Thomsen, and R. D. Belian, Effect of wave-particle interactions on ring current evolution for January 10-11, 1997: Initial results, Geophys. Res. Lett., 25, 2971, 1998.

Jordanova, V. K., C. J. Farrugia, R. M. Thorne, G. V. Khazanov, G. D. Reeves, and M. F. Thomsen, Modeling ring current proton precipitation by electromagnetic ion cyclotron waves during the May 14-16, 1997, storm, J. Geophys. Res, 106, 7, 2001.

Kennel, C. F., Consequences of a magnetospheric plasma, Rev. Geophys., 7 , $379,1969$.

Kennel, C. F., and F. Engelmann, Velocity space diffusion from weak plasma turbulence in a magnetic field, Phys. Fluids, 9, 2377, 1966.

Kennel, C. F., and H. E. Petschek, Limit on stably trapped particle fluxes, J. Geophys. Res., 71, 1, 1966.

Kim, H.-J., and A. A. Chan, Fully adiabatic changes in storm time relativistic electron fluxes, J. Geophys. Res., 102, 22,107, 1997.

Kozyra, J. U., V. K. Jordanova, R. B. Horne, and R. M. Thorne, Modeling of the contribution of electromagnetic ion cyclotron (EMIC) waves to stormtime ring current erosion, in Magnetic Storms, Geophys. Monogr. Ser., vol. 98, edited by B. T. Tsurutani et al., p. 187, AGU, Washington, D. C., 1997.

Li, X., and M. A. Temerin, The electron radiation belt, Space Sci. Rev., 95, 569,2001 
Li, X., I. Roth, M. Temerin, J. R. Wygant, M. K. Hudson, and J. B. Blake, Simulation of the prompt energization and transport of radiation belt particles during the March 24, 1991 SSC, Geophys. Res. Lett., 20, 2423, 1993.

Li, X., D. N. Baker, M. Temerin, T. E. Cayton, E. G. D. Reeves, R. A. Christensen, J. B. Blake, M. D. Looper, R. Nakamura, and S. G. Kanekal, Multi-satellite observations of the outer zone electron variation during the November 3-4, 1993, magnetic storm, J. Geophys. Res., 102, 14,123, 1997.

Lorentzen, K. R., M. P. McCarthy, G. K. Parks, J. E. Foat, R. M. Millan, D. M. Smith, R. P. Lin, and J. P. Treilhou, Precipitation of relativistic electrons by interaction with electromagnetic ion cyclotron waves, J. Geophys. Res., 105, 5381, 2000.

Lyons, L. R., Comments on pitch angle diffusion in the radiation belts, J. Geophys. Res., 78, 6793, 1973.

Lyons, L. R., and R. M. Thorne, Parasitic pitch angle diffusion of radiation belt particles by ion cyclotron waves, J. Geophys. Res., 77, 5608, 1972.

Melrose, D. B., Plasma Astrophysics, Nonthermal Processes in Diffuse Magnetized Plasmas, vol. II, Gordon and Breach, Newark, N.J., 1980.

Meredith, N. P., R. B. Horne, D. Summers, R. M. Thorne, R. H. A. Iles, D. Heynderickx, and R. R. Anderson, Evidence for acceleration of outer zone electrons to relativistic energies by whistler mode chorus, Ann. Geophys., 20, 967, 2002.

Millan, R. H., X-ray observations of $\mathrm{MeV}$ electron precipitation with a balloon-borne germanium spectrometer, Ph.D. dissertation, Dept. of Physics, Univ. of Calif., Berkeley, Calif., 2002.

Perraut, S., R. Gendrin, and A. Roux, Amplification of ion-cyclotron waves for various typical radial profiles of magnetospheric parameters, J. Atmos. Terr. Phys., 38, 1191, 1976

Schulz, M., Particle lifetimes in strong diffusion, Astrophys. Space Sci., 31, 37, 1974.

Steinacker, J., and J. A. Miller, Stochastic gyroresonant electron acceleration in a low-beta plasma, I, Interaction with parallel transverse cold plasma waves, Astrophys. J., 393, 764, 1992.

Stix, T. H., Waves in Plasmas, Am. Inst. of Phys., New York, 1992.

Summers, D., and C.-Y. Ma, A model for generating relativistic electrons in the Earth's inner magnetosphere based on gyroresonant wave-particle interactions, J. Geophys. Res., 105, 2625, 2000 a.
Summers, D., and C.-Y. Ma, Rapid acceleration of electrons in the magnetosphere by fast-mode MHD waves, J. Geophys. Res., 105, 15,887, 2000 b.

Summers, D., R. M. Thorne, and F. Xiao, Relativistic theory of waveparticle resonant diffusion with application to electron acceleration in the magnetosphere, J. Geophys. Res., 103, 20,487, 1998.

Summers, D., R. M. Thorne, and F. Xiao, Gyroresonant acceleration of electrons in the magnetosphere by superluminous electromagnetic waves, J. Geophys. Res., 106, 10,853, 2001.

Thorne, R. M., A possible cause of dayside relativistic electron precipitation events, J. Atmos. Terr. Phys., 36, 635, 1974.

Thorne, R. M., Energetic radiation belt electron precipitation: A natural depletion mechanism for stratospheric ozone, Science, 195, 278, 1977.

Thorne, R. M., and L. J. Andreoli, Mechanisms for intense relativistic electron precipitation, in Exploration of the Polar Upper Atmosphere, edited by C. S. Deehr and J. A. Holtet, p. 381, D. Reidel, Norwell, Mass., 1980.

Thorne, R. M., and R. B. Horne, Modulation of electromagnetic ion cyclotron instability due to interaction with ring current $\mathrm{O}^{+}$during magnetic storms, J. Geophys. Res., 102, 14,155, 1997.

Thorne, R. M., and C. F. Kennel, Relativistic electron precipitation during magnetic storm main phase, J. Geophys. Res., 76, 4446, 1971.

Tsyganenko, N. A., A magnetospheric magnetic field model with a warped tail current sheet, Planet. Space Sci., 37, 5, 1989.

Young, D. T., J. Geiss, H. Balsiger, P. Eberhardt, A. Ghielmetti, and $\mathrm{H}$. Rosenbauer, Discovery of $\mathrm{He}^{2+}$ and $\mathrm{O}^{2+}$ ions of terrestrial origin in the outer magnetosphere, Geophys. Res. Lett., 4, 561, 1977.

Young, D. T., H. Balsiger, and J. Geiss, Correlations of magnetospheric ion composition with geomagnetic and solar activity, J. Geophys. Res., 87, 9077, 1982.

D. Summers, Department of Mathematics and Statistics, Memorial University of Newfoundland, St. John's, Newfoundland, Canada A1C 5S7. (dsummers@math.mun.ca)

R. M. Thorne, Department of Atmospheric Sciences, University of California, P. O. Box 951565, Los Angeles, CA 90095-1565, USA. (rmt@ atmos.ucla.edu) 